\section{Switching off BAFF in the joints can inhibit arthritis development}

Novel therapies for rheumatoid arthritis (RA) that target inflammation locally are highly sought after, as such approaches could avoid systemic immunosuppression. To this end, researchers from Hong Kong successfully targeted B-cell-activating factor (BAFF) in the joints of mice with collagen-induced arthritis $(\mathrm{CIA})$, and showed that silencing this gene suppresses disease development.

Earlier studies have indicated a role for BAFF in the pathogenesis of RA, which prompted Lai Kwan Lam et al. to generate lentiviral vectors expressing short hairpin RNA (shRNA) for BAFF ( $L V$-shBAFF) that, when injected into inflamed joints of CIA mice, resulted in reduced levels of BAFF protein. The percentage of mice developing CIA was reduced by nearly half in the $L V$-shBAFF-treated group in comparison with the control group (who received vectors expressing shRNA for $\beta$-actin); disease onset was delayed and inflammation was milder than in control animals. In mice with established disease, intra-articular LV-shBAFF injection suppressed disease progression and ameliorated joint damage.

BAFF gene silencing resulted in reduced levels of proinflammatory cytokines, including interleukin-17, and reduced numbers of leukocytes, including type 17 T-helper cells, in the joints of treated animals. The lentiviral constructs targeted dendritic cells in the joint tissue, and $L V$-shBAFF-transduced dendritic cells had an immature phenotype and failed to function normally to drive the expansion of type 17 T-helper cells in vitro.

The authors concluded that silencing BAFF in the joints "may prove to be a promising approach for the effective treatment of RA".

Original article Lai Kwan Lam Q et al. (2008) Local BAFF gene silencing suppresses Th17-cell generation and ameliorates autoimmune arthritis. PNAS 105: 14993-14998

\section{Intraperitoneal delivery of nanoparticles targets TNF expression}

Expression of proinflammatory cytokines, including tumor necrosis factor (TNF), by recruited peripheral macrophages has a prominent role in systemic inflammation. Current therapies target TNF with competitive inhibitors, but a study by Howard et al. has demonstrated for the first time that intraperitoneal administration of chitosan nanoparticles containing TNF-specific small interfering RNA (siRNA) could be used to reduce TNF-mediated systemic inflammation at local sites.

In the study, significant knockdown of TNF levels by administration of these nanoparticles was first demonstrated in vitro in stimulated peritoneal macrophages isolated from C57BL/6J mice. In vivo studies then demonstrated the ability of both anti-TNF siRNA modified with 2'-O-methyl nucleotides and unmodified antiTNF siRNA administered by intraperitoneal injection to transfect and silence TNF expression in systemic macrophages. The therapeutic potential of this approach was shown in a type II collagen mouse model of arthritis: peritoneal injection of modified or unmodified anti-TNF siRNA after the onset of arthritis inhibited inflammation and joint destruction and improved survival. Furthermore, administration of anti-TNF siRNA before collagen sensitization delayed onset of the disease, suggesting that anti-TNF RNA could be used as a prophylactic therapy.

Delivery of the therapeutic agent directly to pathogenically active macrophages could overcome the problems of low bioavailability observed with other modes of administration. The results of this study suggest that TNF knockdown via intraperitoneal administration of chitosansiRNA nanoparticles could represent a novel treatment approach for rheumatoid arthritis.

Original article Howard KA et al. (2008) Chitosan/siRNA nanoparticle-mediated TNF- $\alpha$ knockdown in peritoneal macrophages for anti-inflammatory treatment in a murine arthritis model. Mol Ther 17: 162-168

\section{Infliximab superior to conventional treatment for inflammatory eye disease}

Several case studies have demonstrated that tumor necrosis factor inhibitors are effective in the short term for the treatment of uveitis associated with Behçet disease and other immunemediated inflammatory eye diseases. The results of two studies have now shown that infliximab could be superior to conventional immunosuppressive therapy for these conditions. 